Frankfurter elektronische Rundschau zur Altertumskunde 39 (2019)

\title{
Die Landwirtschaft in der Provinz Gallia Narbonensis und die Frage nach dem Sozialstatus der Arbeitskräfte
}

\author{
Thierry Groff
}

\section{Einleitung}

Die Gallia Narbonensis gilt als eine der am stärksten romanisierten Provinzen des Imperium Romanum. Gefördert wurde dieser Umstand sicherlich durch die Nähe zu Italien und die recht frühe Provinzialisierung des Gebietes seit dem 2. Jhdt. v. Chr. ${ }^{1}$ Dabei wurde in der Forschung oft die Frage gestellt, ob ebenso die Agrarstrukturen Italiens, sprich eine auf Sklavenarbeit beruhende Großvillenwirtschaft, in der Narbonensis etabliert wurden. Trotz des eigentlich spärlichen Quellenmaterials ist die Antwort überraschenderweise meistens positiv. Das Ziel dieses Artikels besteht nun darin, diese Aussagen einer kritischen Prüfung zu unterziehen. Hierzu werden insbesondere Inschriften zur Klärung des Sozialstatus der landwirtschaftlichen Arbeitskräfte herangezogen. Ein kurzer Vergleich mit einigen epigraphischen Belegen aus den benachbarten Provinzen und eine Prüfung der Aussagefähigkeit weiterer Quellen, darunter archäologischer und ikonographischer Zeugnisse, soll die Analyse abrunden.

\section{Zum Sozialstatus der Landarbeiter in Südgallien: Die epigraphischen Quellen}

Wie sich die Situation bezüglich eines größeren Sklaveneinsatzes auf den Landgütern der Gallia Narbonensis darstellte, kann eigentlich aufgrund mangelnder Quellen nicht gänzlich geklärt werden. Einige Forscher gehen allerdings davon aus, dass die Umstände mit denen in Italien vergleichbar waren, ${ }^{2}$ wobei u. a. H.-J. Drexhage, H. Konen und K. Ruffing betonen, dass , der massenhafte Gebrauch von Sklaven [...] nördlich der Provinz Gallia Narbonensis [...] weitgehend ausgeschlossen werden [kann]".3 Ähnlich äußert sich auch U. Heimberg und sagt, dass es eine „Sklavenwirtschaft wie in den mediterranen Ländern [...] in Mittel- und Nordgallien und in den germanischen Provinzen nicht [gab] “. ${ }^{4}$ Wie die zitierten Forscher trotz mangelnder Quellen zu solch klaren Aussagen kommen, bleibt fraglich. ${ }^{5}$ Inschriften könnten in dieser Hinsicht eigentlich

\footnotetext{
$1 \mathrm{Zu}$ Geschichte und Entwicklung der Gallia Narbonensis siehe u. a.: Gros 2008; BotermaNN 2005; FREYBERGER 1999; ROMAN 1991; GOUDINEAU 1978.

${ }^{2}$ K. Bradley bringt die Entwicklung der Landwirtschaft in Italien nach dem Hannibal-Krieg wie folgt auf den Punkt: „On this view, from the end of the Hannibalic War onwards (201 BC), the villa system increasingly replaced free labour with slave labour and small-scale subsistence peasant farming with large-scale market-oriented farming. Displaced peasants not consumed by Rome's army streamed to an ever-expanding Rome, where they formed a volatile populace open to the appeals of elite politicians willing to exploit mass discontent for individual political advancement, and the personal enrichment to which through the further expansion of empire office-holding let." S. dazu: BRADLEY 2011, S. 248; und auch K. Ruffing betont, dass ,der wirtschaftliche Aufschwung, den Rom in dieser Epoche ohne jeden Zweifel durchlief, nicht [ohne die Sklaverei] möglich gewesen [wäre]“. S. dazu: RUFFING 2012, S. 91.

${ }^{3}$ DREXHage/Konen/RUfFing 2002, S. 87.

${ }^{4}$ Heimberg 2011, S. $26 f$.

${ }^{5}$ Hypothetisch ist deswegen auch folgende Aussage von N. Brockmeyer: „In Gallien waren die zentralen und südlichen Gebiete am stärksten romanisiert. Hier gab es in den ersten beiden nachchristlichen Jahrhunderten viele villae rusticae, die weitgehend spezialisiert waren und nach den Grundsätzen der
}

http://www.fera-journal.eu 
die zuverlässigsten Zeugnisse darstellen, doch muss beachtet werden, dass einfache Landarbeiter und Sklaven nicht die nötigen Gelder und Mittel besaßen, um sich eine teure Inschrift zu leisten. ${ }^{6}$ Es verwundert demnach kaum, dass Inschriften mit einem Bezug zu Sklaverei und Landwirtschaft in der Gallia Narbonensis selten sind. F. Favory und A. Daubigney konnten bei einer Studie zu den Inschriften Südgalliens lediglich 83 Inschriften erfassen, die mit Sklaven und Freigelassenen auf dem Lande in Verbindung gebracht werden könnten. ${ }^{7}$ Problematisch ist zudem, dass nur sehr wenige dieser Inschriften explizit die Funktion des erwähnten Sklaven oder Freigelassenen nennen, sodass eine Tätigkeit in der Landwirtschaft zwar möglich, so z. B. als actor oder vilicus, aber keineswegs gesichert ist. ${ }^{8}$ Deutlich wird dies z. B. in den Inschriften CIL XII, 619 (FO. Auriol) und CIL XII, 1600 (FO. Die). ${ }^{9}$ Beide Inschriften wurden auf dem Lande, weit entfernt der ehemals städtischen Zentren Südgalliens, entdeckt. Ob es sich bei den Sklaven Alphios und Achilleus allerdings um unfreie Landarbeiter gehandelt hat, geht aus den Inschriften nicht hervor. Inschriften von Sklaven/Freigelassenen, bei denen wohl eine Verbindung zu einer nahegelegenen Villa besteht, konnten ferner in den benachbarten Provinzen entdeckt werden, so z. B. in der Gallia Belgica (CIL XIII, 4199) und in der Germania Superior (CIL XIII, 5312). ${ }^{10}$ Letztgenannte Grabinschrift der Sklavinnen Prima und Araurica wurde in der Nähe der Villa von LiestalMunzach (Schweiz) entdeckt. ${ }^{11}$ Es ist gut möglich, dass die freigelassene Prima als Feldarbeiterin oder im Haushalt tätig war, wobei das gleiche Schicksal wohl auch ihre noch sehr jung verstorbene Schwester erwarten durfte. ${ }^{12}$ Bei der erstgenannten Inschrift aus Hetzerath (Kr. Bernkastel-Wittlich) wurde dem verstorbenen Sklavenkind Primulus

arbeitsteiligen und intensiven, auf Sklavenarbeit beruhenden Landwirtschaftsmethode bewirtschaftet wurden.“ S. dazu: BROCKMEYER 1968, S. 235f.

${ }^{6}$ Diesem Standpunkt schließt sich L. Schumacher an: „In Bezug auf die nordwestlichen Provinzen des Imperium Romanum wird der Sklaveneinsatz von der Forschung sehr zurückhaltend beurteilt. Überwiegend rechnet man mit einem Süd-Nord-Gefälle: Von beträchtlichen Zahlen in Italien über hohe Werte in der römischen Provence (Narbonensis) gehe der Anteil der Sklaven in der Landwirtschaft Galliens immer mehr zurück, für die Gallia Belgica sei kaum noch mit einem Sklaveneinsatz zu rechen. [...] Daß die inschriftliche Überlieferung für diese Problematik keine Entscheidungskriterien bieten kann, brauchen wir nicht zu diskutieren: Einfache Landarbeiter oder Hirten, ob frei oder unfrei, besaßen nicht die Mittel, sich Grabsteine setzen zu lassen, und für Grundbesitzer bestand kaum Veranlassung, ihrer durch eine Stiftung zu gedenken, schon gar nicht unter Erwähnung des Tätigkeitsbereichs." S. dazu: SCHUMACHER 2001, S. 102f.; siehe auch: SAMSON 1989, S. 99-110.

${ }^{7}$ Der Problematik mangelnder Inschriften ist sich auch N. Morley bewusst, der sagt: „Even in Spain and southern Gaul, where it is often assumed that slaves were extensively employed in agriculture, there are few inscriptions from rural contexts or relating to rural occupations; the majority of finds from the countryside refer to personal servants with a few commemorating overseers (vilici). " S. dazu: MORLEY 2011, S. 268; DAUBIGNEY/FAVORY 1972, S. 317f.

${ }^{8}$ Zu Sklaven als actores und vilici siehe: CARLSEN 2010; SCHÄFER 2001; CARLSEN 1995; AUBERT 1994; CARLSEN 1993.

${ }^{9}$ CIL XII, 619 (=AE 2005, 976; = CAG 13/3, S. 781f.): Genio Anni/or(um) Macri / et Liciniani / Alphios ser(vus) / ex voto / pos(u)it lib(ertus). Übersetzung: Dem Genius des Annius Macer und des Annius Licinianus (geweiht). Alphios, Sklave, hat (dieses Denkmal) gemäß seinem Gelübde gestiftet, dies als er die Freiheit erlangte; CIL XII, 1600 (= CAG 26, S. 292; = ILN-07-40): D(is) M(anibus) / Achillei / ser(vi). Übersetzung: Den Totengeistern des Achilleus, Sklave.

${ }^{10}$ MANDER 2013, S. 370; RoymANS 2011, S. 164-169; WIERSCHOWSKi 2001, S. 395; WALSER 1980, Nr. 228.; laut P. Ouzoulias können für die Provinzen Germania Superior, Germania Inferior und Gallia Belgica insgesamt 19 Inschriften erfasst werden, die auf Sklaven im Bereich der Landwirtschaft hindeuten. S. dazu: OuZOulias 2006, S. 224-231.

${ }^{11}$ CIL XIII, 5312 (1. Jhdt. n. Chr.): Prima C(ai) Cotei / lib(erta) ann(orum) XVI et / soror illaeus(!) / Araurica annic(ula) / et mens(ium) VI h(ic) s(itae) s(unt) / patronus po(suit). Übersetzung: Prima, Freigelassene des Caius Coteius, 16 Jahre alt, und ihre Schwester Araurica, ein Jahr und 6 Monate alt, liegen hier begraben. Ihr Patron hat (diesen Grabstein) gesetzt.

${ }^{12}$ zu Frauen auf Landgütern siehe: ROTH 2003.

http://www.fera-journal.eu 
ein Grabstein gesetzt. ${ }^{13}$ Eventuell waren seine Eltern, Sequens und Primula, als Gutsverwalter tätig und hatten somit genügend Geld, um ihrem geliebten Sohn eine Inschrift zu setzen. ${ }^{14}$

Ein Beispiel wie im Falle des Sequens und der Primula kann auch für die Gallia Narbonensis aufgeführt werden: Für L. Buffat u. a. besteht so beispielsweise die Möglichkeit, dass die in der Inschrift CIL XII, 2762 genannten Sklaven Placida und Matutina als Gutsverwalter der bedeutenden Frontonii und Secundii auf dem Gebiet von Nemausus (Nîmes) tätig waren, hatten beide doch immerhin die finanziellen Mittel, sich einen Grabaltar aufzustellen. ${ }^{15}$ Ähnliches gilt auch für eine gewisse Donnia Vera (FO. Coudoux) ${ }^{16}$ deren Gentiliz verrät, dass diese liberta ein Mitglied der Donii war, also einer bedeutenden Familie, die in der Vallée de l'Arc (Provence) mehrere Großgüter besaß. Dass der in ihrer Inschrift angerufene Vitiocelus laut J. Gascou vermutlich eine gallo-römische Fruchtbarkeitsgottheit war, scheint die Möglichkeit einer landwirtschaftlichen Beschäftigung weiter zu erhärten. Dass der Sklave Glycon (FO. Tresques) ebenfalls als actor oder vilicus in der Verwaltung eines Landgutes tätig war, ${ }^{17}$ kann nur vermutet werden. $\mathrm{Zu}$ den wenigen Zeugnissen, welche die Funktion genau angeben, gehört die Inschrift eines aus Grenoble stammenden actor namens Fronto. ${ }^{18}$ Dabei nimmt F. Sudi-Guiral an, dass es sich nicht um einen öffentlichen, sondern um einen in der privaten Gutsverwaltung tätigen Sklaven gehandelt hat. Weitere, eindeutig im Agrarsektor beschäftigte actores sind für die Narbonensis nicht bezeugt, dafür aber für die benachbarten Provinzen Aquitania (FO. Saint-Pe-d'Ardet) und Lugdunensis (FO. Lyon). ${ }^{19}$ Eine epigraphische Angabe einer praedia oder eines fundus/locus kann die

\footnotetext{
${ }^{13}$ CIL XIII, 4199 (2./3. Jhdt. n. Chr.): D(is) M(anibus) / Primuli infa/ntis Sequen/tis et Primul(a)e / filio servo. Übersetzung: Den Totengeistern des Verstorbenen. Primulus, Sohn des Sequens und der Primula, ein Sklavenkind.

${ }^{14}$ ROYMANS 2011, S. 164-169;

15 CIL XII, 2762 (= CAG 30/3, S. 709; 2./3. Jhdt. n. Chr.): D(is) M(anibus)//Plac/I / vi $<\mathrm{v}>\mathrm{a} /$ sibi / p(o)s(ui)t// Matu/tini / Placi/da co/ntub/erna/li o $<\mathrm{p}>$ ti/mo p(o)s(ui)t. Übersetzung: Den Totengeistern der Placida. Sie hat diesen Altar zu Lebzeiten für sich selbst aufgestellt // Den Totengeistern des Matutinus. Placida hat diesen Altar für den besten Lebensgefährten aufgestellt; BUFFAT/CHRISTOL u. a. 2002, S. 223-225 und 236-238.

${ }^{16}$ AE 1992, 1180: Vitiocelo / Donnia G(ai!) / liberta / Vera / v(otum) s(olvit) l(ibens) m(erito). Übersetzung: Für Vitiocelus! Donnia Vera, Freigelassene des Caius, hat ihr Gelübde gerne und wie es sich gehört (oder: gut und gerne) eingelöst.; Ein Mitglied der Donii, L. Donnius Flavus, war Priester des Kaiserkultes und hat den nach ihm benannten Pont Flavien gestiftet. Bezeugt wird dieser Umstand von der Inschrift CIL XII, 647. Die Brücke befindet sich nur $17 \mathrm{~km}$ vom Fundort der Donnia-Inschrift entfernt; LEVEAU 2002, S. 77; GASCOU 1992, S. 133-136.

${ }^{17}$ CIL XII, 2753 (= CAG 30/3, S. 711; 2./3. Jhdt. n. Chr.): Iovi / O(ptimo) M(aximo) / dedi/cavit / Gly[c]/on. Übersetzung: Für Iupiter Optimus Maximus. Glycon hat dies geweiht; CAVALIER 2005, S. 19. ${ }^{18}$ CIL XII, 2250 (= CAG 38/1, S. 89; = ILN-05-02-387; 2./3. Jhdt. n. Chr.): D(is) M(anibus) / Frontonis / actoris huius / loci Materna / coniugi karissimo / [Ph]ilusa patri dul/cissimo / facien/dum curavit(!) / et Eudrepites / filius parenti / optimo sub ascia / d[edicav(erunt?)]. Übersetzung: Den Totengeistern des Fronto, Verwalter dieses Landgutes. Materna ihrem liebsten Gatten, Philusa ihrem sehr geliebten Vater, haben (diesen Grabstein) errichtet und Eudrepites, sein Sohn, hat ihn dem besten aller Väter unter der ascia geweiht.; SUDI-GUIRAL 2008, S. 407.

${ }^{19}$ CIL XIII, 66 (=CAG 31/2, S. 426; = AE 1888, 143; = AE 2009, 856; Datierung unbekannt): I(ovi) O(ptimo) M(aximo) / Sabini/anus ser(vus) ac/tor Paulin/iani n(os)t(ri) / v(otum) s(olvit) 1(ibens) m(erito). Übersetzung: Dem Jupiter Optmius Maximus. Sabinianus, Sklave und Aufseher/Verwalter im Dienste unseres Paulinianus. Er hat sein Gelübde gern und wie es sich gehört (oder: gut und gerne) eingelöst.; CIL XIII, 2243 (= CAG 69/2, S. 420; 2. Jhdt. n. Chr.): Text: D(is) M(anibus) / Primitivi ac/toris praedi/orum horum / vivus sibi pos/terisque suis / fecit et sub as/cia dedicavit. Übersetzung: Den Totengeistern des Verstorbenen Primitivus, Aufseher/Verwalter dieses Anwesens, der, zu seinen Lebzeiten, dieses Grab für sich und seine Nachkommen gestiftet und unter der ascia geweiht hat; S. dazu auch: CARLSEN 1995, S. 137f.
}

http://www.fera-journal.eu 
direkte Verbindung zu einem Landgut plausibel machen, so geschehen in der südgallischen Inschrift des Sklaven Niger (FO. Allan): ${ }^{20}$ Aus seiner den Matronen gewidmeten Inschrift geht nämlich hervor, dass er als cellarius (Kellermeister) auf dem Landgut Anarias tätig war. Besitzer dieser Domäne war ein gewisser C. Atrius Silius. Ob der aus Arles stammende dispensator Peregrinus (FO. Arles) in den landwirtschaftlichen Finanzgeschäften seines Herrn beschäftigt war, muss dagegen wiederum offenbleiben. ${ }^{21}$ Ebenfalls unklar ist, ob die Freigelassene Rusticania auf einem Gutshof in der Nähe des heutigen Perpignan beschäftigt war (FO. Théza), doch könnte die Ähnlichkeit ihres Namens mit dem Wort rustica (Bäuerin) zumindest eine Andeutung dafür sein. ${ }^{22}$ Abschließend können auch Inschriften von Sklaven und Freigelassenen, die den Göttern Silvanus, Mars, Jupiter, Ceres oder Juno eine Weihung dargebracht haben, als Indizien für eine Beschäftigung in der Landwirtschaft dienen, da diese Götter bei Landwirten, Landarbeitern und Viehhütern als Schutzgottheiten sehr beliebt waren. ${ }^{23}$ Beispielhaft sei hier die Inschrift CIL XII, 1834 (FO. Vienne) angeführt, die angibt, dass der Sklave Primigenius dem Gott Silvanus ein Gelübde dargebracht hat. ${ }^{24}$ Ebenfalls dem Silvanus hat der Freigelassene C. Iulius Thallus (CIL XII, 1521; FO. Aubignosc) eine Weihinschrift gesetzt, ${ }^{25}$ wobei P. Leveau vermutet, dass dieser libertus in Verbindung zu einer der großen Villen im Durance-Tal bei Sisteron (Segustero) stand. ${ }^{26}$

\section{Wirtschaftliche Blüte dank hohem Sklaveneinsatz? Analyse und Ausblick}

Die aufgeführten Inschriften zeigen, dass servile Arbeitskräfte mit hoher Wahrscheinlichkeit auf den villae rusticae Südfrankreichs eingesetzt wurden, wobei epigraphische Zeugnisse aus den benachbarten Provinzen diese Vermutung zudem erhärten. Wie hoch ihre Zahl war, kann jedoch nicht eindeutig beantwortet werden. Auch gilt es zu beachten, dass die aufgeführten Inschriften aller Voraussicht nach von Unfreien gesetzt wurden, die sich die Finanzierung eines Grabsteines oder einer Weihinschrift überhaupt

\footnotetext{
${ }^{20}$ ILGN 257(= AE 1907, 40; = CAG 26, S. 151f.; Datierung unbekannt): Matris(!) V[ic]tricibus / [v(otum)] s(olvit) [1(ibens)] m(erito) / Niger C(ai) Satri Sili (servus) / cellariu[s] Anariate / d(e) [s(uo)] d(at). Übersetzung: Den siegreichen Matres! Niger, Sklave des Caius Satrius Silus, Kellermeister auf dem Anwesen Anarias, hat sein Gelübde gerne und wie es sich gehört (oder: gut und gerne) eingelöst. Er gab/stiftete dies aus eigenen Geldern.

${ }^{21}$ CIL XII, 856 (= CAG 13/5, S. 510; 2. Jhdt. n. Chr.): Peregrino / Antistiae Piae / dispensatori / Antistia Piae liberta / Cypare contubern(ali) / pientissimo. Übersetzung: Für Peregrinus, Schatzmeister/Verwalter der Antistia Pia. Antistia Cypare, Freigelassene der Antistia, ihrem äußerst liebevollen Lebensgefährten.; Laut J.-J. Aubert konnten die im Geldgeschäft tätigen dispensatores auch die Finanzgeschäfte der Landgüter ihrer domini verwalten. Dispensatores waren i. d. R. immer Sklaven und genossen die gleiche Sozialstellung und die gleichen Privilegien wie die vilici und actores. S. dazu: AUBERT 1994, S. 196-199.

${ }^{22}$ CIL XII, 5363 (= CAG 66, S. 596; 2./3. Jhdt. n. Chr.); D(is) M(anibus) / Rusticaniae L(uci) / lib(ertae) CO[---] / [vi]xi[t anno]s / CXI [men]ses [---]. Übersetzung: Den Totengeistern der Rusticania Corana(?), Freigelassene des Lucius. Sie lebte (?) Jahre und 111 Monate?

${ }^{23}$ So bringt u. a. Cato diese Gottheiten in seinem Werk De agri cultura mit Opfergaben und Kulthandlungen auf Landgütern in Verbindung. S. dazu: Cat. agr. 92.

${ }^{24}$ CIL XII, 1834 (=CAG 38/3, S. 387; = ILN-05-01-20; Datierung unbekannt): Text: Silvano Au[g(usto)] / Primigeniu[s] / Victoris s(ervus) v(otum) [s(olvit)]. Übersetzung: Dem erhabenen Silvanus. Primigenius, Sklave des Victorius, hat sein Gelübde gern und wie es sich gehört (oder: gut und gerne) eingelöst. ${ }^{25}$ CIL XII, 1521 (= CAG 4, S. 79; Datierung unbekannt): Silvano / C(aius) Iul(ius) / Thallus / ex voto. Übersetzung: Für Silvanus. Caius Iulius Thallus, aus einem Gelübde heraus; LEVEAU 2002, S. 65f. und 77.

${ }^{26}$ Morley 2011, S. 268f.; BufFAt/Christol 2002, S. 219-227 und 236-238; LEVEAU 2002, S. 65f. und 77; Aubert 1994, S. 137f.; Kolendo 1993, S. 267-274; SAMSON 1989, S. 100f.; Belova 1987, S. $107-$ 115; DAUBIGNEY/FAVORY 1972, S. 317f., 323-329 und 335-337.
}

http://www.fera-journal.eu 
leisten konnten. Einfache Sklavenarbeiter, die, wenn überhaupt, die Mehrzahl der unfreien Landarbeiter gestellt haben, sind demnach kaum zu erfassen. Eine hohe Präsenz von Sklaven und Freigelassenen ist aufgrund zahlreicher Inschriften zumindest in den Städten Südgalliens bezeugt, dies v. a. im Gewerbe und im Handel.$^{27}$ Daneben sprechen der hohe Grad der Romanisierung, die hohe Zahl italischer Einwanderer sowie das Aufblühen der Villenwirtschaft in Südgallien dafür, dass ein größerer Arbeitseinsatz von Unfreien in der Agrarwirtschaft stattgefunden haben könnte. ${ }^{28}$ Neben kleineren gab es dabei auch sehr große Landgüter, wobei die Schafzucht in der Crau sowie der für die Narbonensis sehr bedeutende Anbau von Wein und Getreide sicherlich eine größere Anzahl an Arbeitern und Hirten benötigte. ${ }^{29}$ Viele Landgüter befanden sich zudem in der Nähe von Städten, also genau dort, wo eine erhöhte Anzahl an Sklaven und Freigelassenen anzutreffen war. Auch gilt es zu beachten, dass die Besitzer der größeren Villen teilweise in den Städten lebten und über genügend Gelder verfügten, um sich Sklaven anzuschaffen. Weil darüber hinaus und anders als für Italien, Spanien oder Nordafrika literarische Zeugnisse zur Sklaverei in Gallien fehlen, können neben Inschriften nur noch materielle Hinterlassenschaften die Anwesenheit von Unfreien in der Landwirtschaft Südgalliens bezeugen. ${ }^{30}$ Dazu gehören Fuß- oder Halsketten, die Reste serviler Behausungen/ergastulae, Unterkünfte für den vilicus sowie ikonographische Darstellungen. Bildnisse von vermeintlichen Sklaven und Dienern auf Grabsteinen kennt man u. a. von den sogenannten Totenmahlszenen bzw. Reitergrabsteinen. ${ }^{31}$ Ein

${ }^{27}$ F. Favory und A. Daubigney haben fast 6000 Inschriften für die Narbonensis gesammelt und untersucht. 4826 Inschriften beziehen sich dabei auf das urbane Milieu, von denen wiederum 863 mit Sklaven/Freigelassenen in Verbindung gebracht werden können (17,5\%). Aus dem ländlichen Raum wurden nur 1054 Inschriften erfasst, wobei 83 auf Unfreie hindeuten; Laut M. Gayraud haben Unfreie 30,5\% der Gesamtbevölkerung von Narbonne ausgemacht, in Béziers waren es laut M. Clavel 17,5\%. S. dazu: GrofF 2020, S. 142; GAYRAUD 1981, S. 473; DAUBIGNEY/FAVORY 1972, S. 317-320 und 326-328; CLAVEL 1970, S. 589.

${ }^{28}$ Zur hoch entwickelten Landwirtschaft Südgalliens in gallo-römischer Zeit siehe u. a.: BUFFAT 2011; BufFat 2010; TRÉMENT 2010; OUZOULIAS 2010; LEVEAU 2001; FERDIÈRE 1988.

${ }^{29}$ Beispielhaft kann auf die Untersuchung der Villenwirtschaft durch I. Bermond. und C. Pellecuer in der Region des Etang de Thau (Hérault) verwiesen werden. Beide Forscher haben herausgefunden, dass es hier in römischer Zeit neben kleineren Gutshöfen auch sehr bedeutende villae rusticae gab, die über mehrere hundert Hektar Land verfügten (u. a. die Villen von Prés-Bas, Mas de Lavit, Mèze und Yeuses). Auf den Ländereien, die neben flachen Ebenen auch hügeliges Terrain umfassten, wurden neben Wein und Getreide auch Oliven angebaut. Diese Umstände sind sicherlich auch zu einem großen Teil auf die anderen Regionen Südgalliens übertragbar. S. dazu: BERMOND/PELLECUER 1997, S. 67-81; zur hoch entwickelten Weinwirtschaft in Südgallien siehe u. a.: GOMEZ 2012; LAUBENHEIMER 2009; BRUN 2003; BRUN 2001; BUFFAT 2001; BOISSINOT 2001; JUNG 2001; LAUBENHEIMER 2001; LAUBENHEIMER 1990; LAUBENHEIMER 1989; laut Cato benötigte man zur Ausstattung von 100 Joch Rebland 14 Arbeiter: Cato agr. 13, 1; zur Schafzucht in der Crau siehe u. a. LEGUILlOUX 2003; BADAN/CONGÉS/BRUN 1995.

${ }^{30}$ Auf der iberischen Halbinsel, wo in römischer Zeit u. a. die Ölproduktion der Baetica industrielle Züge angenommen hatte, haben sich seit spätrepublikanischer Zeit große Latifundien entwickelt, auf denen vermutlich zahlreiche Sklaven nach italischem Vorbild eingesetzt wurden. Neben einigen Inschriften (CIL II, 1980) zeigen auch einzelne Schriftquellen, darunter Caesars Commentarii de Bello Civili (Caes. civ. 1, 51) und Appians Bürgerkriege (App. B. C. 2, 103), dass es in Spanien zahlreiche Sklaven gab. Außerdem hat Varro längere Zeit in Spanien gelebt, sodass der Schriftsteller wohl einiges an seiner dort gemachten Erfahrung in seine Rerum rusticarum libri tres mit eingebracht hat. Gleiches gilt für Columella, haben doch einige seiner Familienmitglieder als Gutsbesitzer auf der iberischen Halbinsel gelebt; BLÁZQUEZ/LE RouX 1972, S. 634-639; literarische Zeugnisse zu Sklaven in der Landwirtschaft Nordafrikas bieten uns Apuleius, der über die Ausstattung großer Latifundien berichtet (Apul. apol. 93), Sallust in seinem Bellum Iugurthinum (Sall. Iug. 44, 5) und Petronius, der in seinen Satyrica von einem imaginären Gutsbesitzer spricht, der in Numidien so viele Sklaven besessen haben soll, dass er mit diesen Karthago hätte erobern können (Petr. 117, 8); GSELL 1932, S. 397-415.

${ }^{31}$ Für die Narbonensis ergibt sich aber das Problem, dass es nur sehr wenige solcher bildlichen Darstellungen gibt; zu den Reitergrabsteinen und Totenmahlszenen siehe: NoELKE 2005, S. 155-242;

http://www.fera-journal.eu 
schönes, aus Südfrankreich stammendes Beispiel von der Darstellung möglicher Sklaven, kommt aus Saint-Romain-en-Gal. Dort ist auf einem Mosaik zu erkennen, wie drei Arbeiter die geernteten Trauben im Maischbecken zertreten (Abb. 1). Allerdings muss es sich bei diesen Arbeitern nicht unbedingt um Sklaven handeln, sondern eventuell um Tagelöhner oder Saisonarbeiter, sodass die Grenzen bei der Interpretation bildlicher Darstellungen bezüglich der Sklaverei hier schnell erkennbar werden. Fesseln wurden zum Teil in Britannien sowie in Mittel- und Nordgallien gefunden, wobei auch zwei solcher Gegenstände in Südgallien entdeckt wurden, darunter in einer Grotte bei Tarascon-sur-Ariège (Dép. Ariège) und in einer Abfallgrube in Millau (Dép. Aveyron). $\mathrm{Ob}$ diese Fesseln aber tatsächlich von unfreien Landarbeitern oder einem entflohenen Sklaven aus einer Mine/Stadt getragen wurden, ist unbekannt, da sie ebenso zum Festhalten von Tieren oder zur Bestrafung eines Lohnarbeiters genutzt worden sein könnten. ${ }^{32}$ Wohngebäude, die als Sklavenbehausungen oder ergastulae gedient haben könnten, wurden ebenfalls nur vereinzelt in Germanien, in Mittel- und Nordgallien, in Aquitanien, in der Gallia Narbonensis (Villa von Chiragan; Dép. Haute-Garonne) sowie im direkt benachbarten Spanien entdeckt. Dabei könnten diese Gebäude aber auch ganz einfach als Unterkünfte für Lohn- oder Saisonarbeiter bzw. als Abstellkammern gedient haben, weshalb bei der Deutung dieser Hinterlassenschaften keine vorschnellen Schlüsse gezogen werden sollten. ${ }^{33} \mathrm{Ob}$ sich in Südgallien demnach ein Sklavenhaltersystem nach italischem Vorbild ausgebildet hat, können archäologische Überreste ebenso wenig klären, wie die eigentlich zuverlässigeren Inschriften. Die Aussage von N. Morley, dass ,neither epigraphic nor archeological evidence can rule out the possibility that slave labour was extensively employed in the provinces" ${ }^{34}$ ist deswegen ohne weiteres auf die Umstände in der Gallia Narbonensis übertragbar, wobei es in Südfrankreich sicherlich auch eine hohe Quote an freien Pächtern und unabhängigen Kleinbauern gab. Und auch wenn weitere Forschungen das zukünftige Gesamtbild erweitern mögen, so scheint es doch kaum möglich, je eine zuverlässige Aussage zur genauen Anzahl an Unfreien in der Landwirtschaft Südgalliens zu tätigen. ${ }^{35}$

SCHUMACHER 2001, S. 82-90 und 190; ANDERSON 1984, S. 25-34; Schumacher betont, dass man bei der Interpretation der Bilder aufpassen muss. Oft sind Kinder und Sklaven, die man an ihrer Größe bzw. an ihrer dargestellten Handlung erkennen mag, nicht voneinander zu unterschieden. Daneben stellt sich auch die Frage, inwiefern diese Darstellungen der Realität entsprechen, da viele Verstorbene eventuell einen Sklaven aus Statusgründen abbilden ließen.

${ }^{32} \mathrm{Zu}$ Sklavenfesseln aus der Eisenzeit und aus der römischen Antike siehe: THOMPSON 1993, S. 57-168.

${ }^{33}$ Die Interpretation, dass es sich bei einem Gebäude um ein ergastulum gehandelt haben könnte, gründet sich i. d. R. auf zwei Komponenten: erstens auf den Aufbau und die Lage des Gebäudes und zweitens auf die Tatsache, dass eine größere Anzahl von Werkzeugen in direkter Nähe entdeckt wurden. Dass eine sichere Interpretation aber schwierig ist, soll folgende Aussage von N. Belova zeigen: „In einigen Fällen zeugt der Charakter der auf dem Landgut errichteten Wirtschaftsgebäude oder die Häufung einer großen Zahl von Arbeitsgegenständen von der Verwendung der Sklavenarbeit. Auf dem Gebiet der Parisier in der Gallia Lugdunensis entdeckte man z. B. zwischen den Überresten der Villa Guiry-Gadancourt einen kleinen Bau, der für die Aufbewahrung des Produktionsinventars bestimmt war. Außerdem fand man ein Wohngebäude für die auf dem Landgut beschäftigten Arbeiter." Dass diese Arbeiter Sklaven gewesen sein sollen, geht aus der Aussage Belovas aber definitiv nicht hervor, da eindeutige Beweise schlicht fehlen. S. dazu: BELOVA 1987, S. 110.

${ }^{34}$ MORLEY 2011, S. 273.

${ }^{35}$ Groff 2020, S. 140; Morley 2011, S. 269-274; RoyMAns 2011, S. 169-175; FeUGÈre 2008, S. 14f.; Aubert 1994, S. 123-125; PleKET 1990, S. 100-102; SAMSON 1989, S. 100-107; BElOVA 1987, S. 109111; MacMullen 1987, S. 367-371; Labrousse 1978, S. 390; LABrousse 1974, S. 457; DaUBIGNEY/FAVORY 1972, S. 317f. und 323-329; BROCKMEYER 1968, S. 204-245 (geht auch auf Africa, Ägypten, Spanien, Griechenland und den Donauraum ein); PIGANIOL 1959, S. 276-279; MiTARD 1958, S. 266280 . 
Frankfurter elektronische Rundschau zur Altertumskunde 39 (2019)

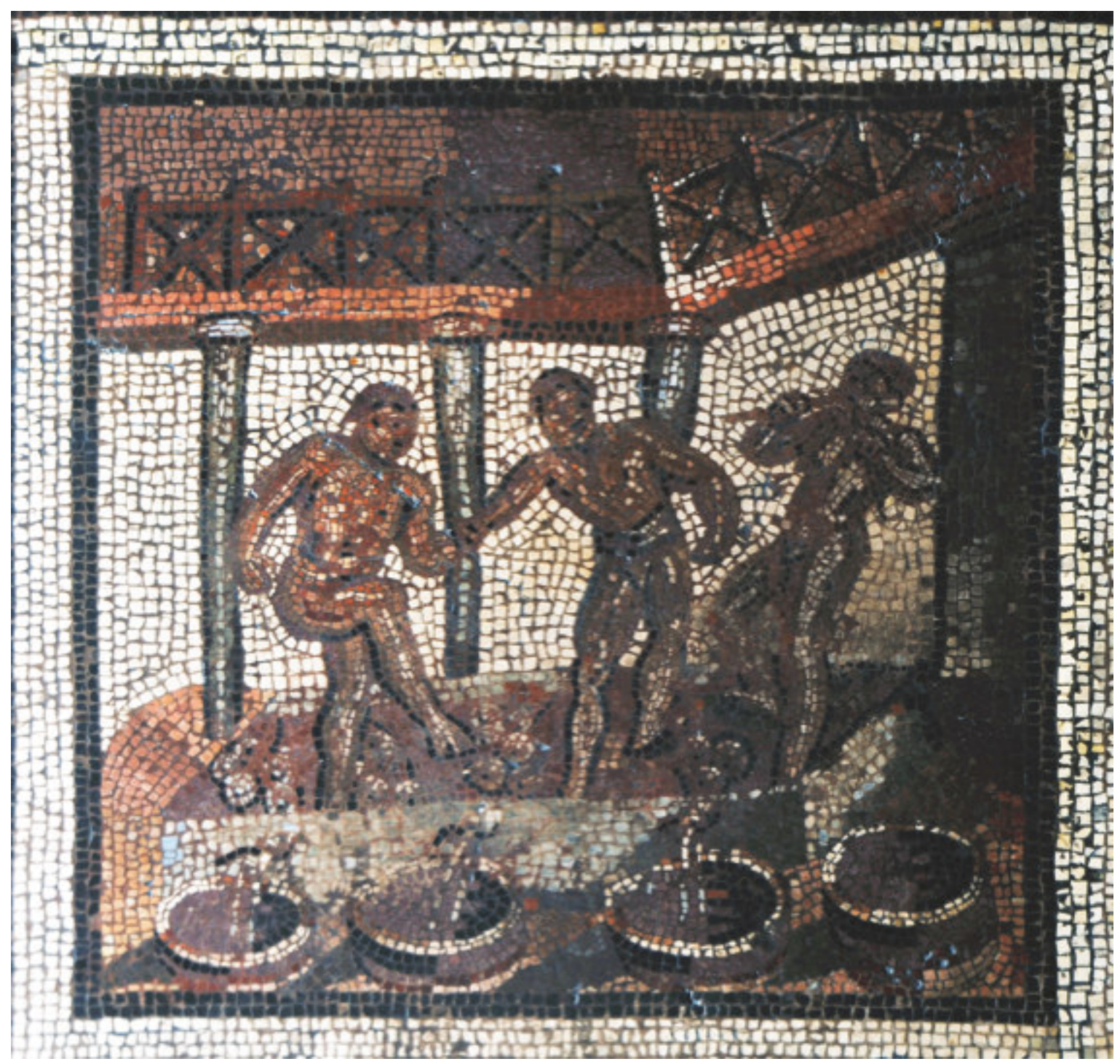

Abb 1: Detail eines Mosaiks aus Saint-Romain-en-Gal, welches Sklaven (?) beim Zertreten von Trauben zeigt (ㄷ F. Lontcho - Musée Archéologique National - SaintGermain-en-Laye) 


\section{Siglen}

AE: $\quad$ L'Année Épigraphique

AJ: $\quad$ The Archeological Journal

BICS: $\quad$ Bulletin of the Institute of Classical Studies

BJ: $\quad$ Bonner Jahrbücher

CAG: $\quad$ Carte Archéologique de la Gaule

CIL: $\quad$ Corpus Inscriptionum Latinarum

ILGN: Inscriptions Latines de Gaule Narbonnaise

MCV: $\quad$ Mélanges de la Casa Velàzquez

RANarb: Revue Archéologique de Narbonnaise

REA: Revue des Études Anciennes

ZPE: $\quad$ Zeitschrift für Papyrologie und Epigraphik

\section{Literaturverzeichnis}

Adjadj, F. (Hrsg.): Carte Archéologique de la Gaule 38/3. Vienne, Paris 2013.

Anderson, A. S.: Roman Military Tombstones, Aylesbury/Bucks (UK) 1984.

AUBERT, J.-J.: Business managers in ancient Rome. A Social and Economic Study of Institores (200 B.C. - A.D. 250), Leiden/New York/Köln 1994.

BADAN, O./CONGÉS, G./BRUn, J-P.: Les bergeries romaines de la Crau d'Arles. Les origines de la transhumance en Provence, in: Gallia 52 (1995), S. 263-310.

Belova, N. N.: Die Sklaverei im römischen Gallien, in: STAERMAN, E. M. u. a. (Hrsg.): Die Sklaverei in den westlichen Provinzen des Römischen Reiches im 1.-3. Jahrhundert, Stuttgart 1987, S. 103-146.

BÉRARD, G.: Carte Archéologique de la Gaule 4. Les Alpes-de-Haute-Provence, Paris $2013^{2}$.

Bermond, I./Pellecuer, Ch.: Recherches sur les occupations du sol dans la région de l'étang de Thau (Hérault). Apport à l'étude des villae et des campagnes de Narbonnaise, in: RANarb 30 (1997), S. 63-84.

BLÁZQueZ, J-M./LE RouX, P.: L'esclavage dans les exploitations agricoles de l'Hispania romaine, in: MCV 8 (1972), S. 634-639.

BoIssinOt, Ph.: L'archéologie des vignobles antiques du sud de la Gaule, in: Gallia 58 (2001), S. 45-68.

BotermanN, H.: Wie aus Galliern Römer wurden. Leben im Römischen Reich, Stuttgart 2005.

Bradley: K.: Slavery in the Roman Republic, in: BrAdLEY, K./CARTLEDGE, P. (Hrsg.): The Cambridge World History of Slavery. Volume I. The Ancient Mediterranean World, Cambridge 2011, S. 241-264. 
BROCKMEYER, N.: Arbeitsorganisation und ökonomisches Denken in der Gutswirtschaft des römischen Reiches, Bochum 1968.

BRUN, J-P.: La viticulture antique en Provence, in: Gallia 58 (2001), S. 69-89.

BRUN, J-P.: Le vin et l'huile dans la Méditerranée antique. Viticulture, oléiculture et procédés de fabrication, Arles 2003.

Buffat, L. u. a.: La viticulture antique en Languedoc-Roussillon, in: Gallia 58 (2001), S. 91-111.

BUFFAT, L. u. a.: Le problème d'interprétation des établissements ruraux de grandes dimension. Quelques cas en Languedoc, in: RANarb 35 (2002), S. 199-239.

Buffat, L.: Fermes et villas en Gaule Narbonnaise, in: Ouzoulias, P./TranOY, L. (Hrsg.): Comment les Gaules devinrent romaines, Paris 2010, S. 177-188.

BufFAT, L.: L'économie domaniale en Gaule Narbonnaise, Lattes 2011.

CARLSEN, J.: The vilica and roman estate management, in: SANCISI-WEERDENBURG, H. u. a. (Hrsg.): De Agricultura. In Memoriam Pieter Willem de Neeve (19451990), Amsterdam 1993, S. 197-205.

CARLSEN, J.: Vilici and roman estate managers until AD 284, Rom 1995.

CARLSEN, J.: Recruitement and training of Roman Estate Managers in a comparative perspective, in: BICS 109 (2010), S. 75-90.

CAVAliER, O.: La collection d'inscriptions gallo-grecques et latines du Musée Calvet, Paris 2005.

Clavel, M.: Béziers et son territoire dans l'antiquité, Paris 1970.

DAubigney, A. / FAvory, F.: L'esclavage en Narbonnaise et Lyonnaise d'après les sources épigraphiques, in: Actes du colloque 1972 sur l'esclavage. Bésançon 23 mai 1972, Bésançon 1972, S. 315-388.

Drexhage, H-J./Konen, H./Ruffing, K.: Die Wirtschaft des Römischen Reiches (1.3. Jahrhundert). Eine Einführung, Berlin 2002.

FERDIÈre, A.: Les campagnes en Gaule Romaine. Tome 2, Les techniques et les productions rurales en Gaule (52 av. J.-C. - 486 ap. J.-C.), Paris 1988.

FeugÈRE, M.: Maîtres et esclaves dans l'économie de la Gaule méridionale, in: Instrumentum 27 (2008), S. 14-16.

Freyberger, B.: Südgallien im 1. Jahrhundert v. Chr. Phasen, Konsequenzen und Grenzen römischer Eroberung 125-27/22 v. Chr. (Geographica Historica, 11), Stuttgart 1999.

GASCOU, J.: Une nouvelle divinité gallo-romaine d'après une inscription de Coudoux (Bouches-du-Rhône), in: ZPE 93 (1992), S. 133-136.

GAYRAUD, M.: Narbonne antique des origines à la fin du IIIe siècle, Paris 1981.

Gomez, E.: La viticulture, in: Ugolini, Daniela/Olive, Christian (Hrsg.): Carte Archéologique de la Gaule 34/4. Béziers, Paris 2012, S. 156f.

Goudineau, Ch.: La Gaule Transalpine, in: Nicolet, C. (Hrsg.): Rome et la conquête du monde méditerranéen, Bd. 2. Genèse d'un empire, Paris 1978, S. 679-699. 
Groff, T.: Die Minen und Steinbrüche der Provinz Gallia Narbonensis. Eine Untersuchung zu Abbau, Nutzung und Status der freien und unfreien Arbeitskräfte im römischen Südgallien, in: MBAH 37 (2020), S. 127-155 (in Vorbereitung).

Gros, P.: Gallia Narbonensis. Eine römische Provinz in Südfrankreich, Mainz 2008.

GSELL, S.: Esclaves ruraux dans l'afrique romaine, in: Mélanges Gustave Glotz. Bd. 1, Paris 1932, S. 397-415.

HEIMBERG, U.: Villa rustica. Leben und Arbeiten auf römischen Landgütern, Darmstadt 2011.

JUNG, C. u. a.: La viticulture antique dans le Tricastin (moyenne vallée du Rhône), in: Gallia 58 (2001), S. 113-128.

Kolendo, J.: La religion des esclaves dans le De agricultura de Caton, in: Religion et anthropologie de l'esclavage et des formes de dépandance (Actes des colloques du Groupe de recherche sur l'esclavage dans l'antiquité, Bd. 20), Bésançon 1993, S. 267-274.

Kotarba, J./Castellvi, G./Mazière, F. (Hrsg.): Carte Archéologique de la Gaule 66. Les Pyrénées-Orientales, Paris 2007.

Labrousse, M.: Circonscription de Midi-Pyrénées, in: Gallia 32/2 (1974), S. 453-500.

Labrousse, M.: Circonscription de Midi-Pyrénées, in: Gallia 36/2 (1978), S. 389-430.

LAUBENHEIMER, F.: Le vin gaulois, in: REA 91 (1989), S. 5-22.

LAubenheimer, F.: Le temps des Amphores en Gaule. Vins, huiles et sauces, Paris 1990.

LAUBENHEIMER, F.: Le vin gaulois de Narbonnaise exporté dans le monde romain, sous le Haut-Empire. In: LAUBENHEIMER, F. (Hrsg.): 20 ans de recherche à Sallèles d'Aude. Colloque des 27-28 septembre 1996, Bésançon 2001, S. 51-66.

LAUBENHEIMER, F./SCHMitT A.: Amphores vinaires de Narbonnaise, Production et grand commerce. Création d'une base de données géochimiques des ateliers (Travaux de la Maison de l'Orient et de la Méditerrannée, 51), Lyon 2009.

LeguillouX, M.: Les bergeries de la Crau. Production et commerce de la Laine, in: Revue Archéologique de Picardie 1-2 (2003), S. 339-346.

Le Mer, A-C/Chomer, C. (Hrsg.): Carte Archéologique de la Gaule 69/2. Lyon, Paris 2007.

LEVEAU, Ph.: Veränderungen der Landwirtschaft Südgalliens im Zuge der römischen Herrschaftsausübung, in: HERZ, P./WALDHERR, G. (Hrsg.): Landwirtschaft im Imperium Romanum (Pharos - Studien zur griechisch-römischen Antike), St. Katharinen 2001, S. 135-155.

LeVEAU, Ph.: L'habitat rural dans la Provence antique: Villa, vicus et mansion. Etudes de cas, in: RANarb 35 (2002), S. 59-92.

MAcMullen, R.: Late Roman Slavery, in: Historia 36/3 (1987), S. 359-382.

MANDER, J.: Portraits of Children on Roman funerary monuments, Cambridge 2013. 
MitARD, P-H.: La villa gallo-romaine de Guiry-Gadancourt (Seine-et-Oise), in: Gallia $16 / 2$ (1958), S. 266-280.

Morley, N.: Slavery under the Principate, in: Bradley, K./CARTledge, P. (Hrsg.): The Cambridge World History of Slavery. Volume I. The Ancient Mediterranean World, Cambridge 2011, S. 265-286.

NOELKE, P.: $\mathrm{Zu}$ den Grabreliefs mit Darstellung des convivium coniugale im römischen Germanien und im benachbarten Gallien, in: BJ 205 (2005), S. 155-242.

Ouzoulias, P.: L'économie agraire de la Gaule. Aperçus historiographiques et perspectives archéologiques, Dissertation an der Université Franche-Compté Bésançon, Frankreich 2006.

OuZOULIAS, P.: Les campagnes gallo-romaines. Quelle place pour la villa?, in: Ouzoulias, P./Tranoy, L. (Hrsg.): Comment les Gaules devinrent romaines, Paris 2010, S. 189-210.

Pelletier, A. u. a. (Hrsg.): Carte Archéologique de la Gaule 38/1. L'Isère, Paris 1994.

Piganiol, A.: Circonscriptions de Paris (Nord), in: Gallia 17/2 (1959), S. 267-292.

Planchon, J./Bois, M./Conjard-Réthoré, P. (Hrsg.): Carte Archéologique de la Gaule 26. La Drôme, Paris 2010.

Pleket, H. W.: Wirtschaft, in: VitTinghoff, F. (Hrsg.): Europäische Wirtschafts- und Sozialgeschichte der römischen Kaiserzeit, Stuttgart 1990, S. 25-160.

Provost, M. u. a. (Hrsg.): Carte Archéologique de la Gaule 31/3. Le Gard, Paris $2016^{2}$.

Roth, U.: The female slave in Roman agriculture. Changing the default, Nottingham 2003.

Rothé, M-P./TréZINY, H.: Carte Archéologique de la Gaule 13/3. Marseille et ses alentours, Paris 2005.

Rothé, M-P./Heijmans, M. (Hrsg.): Carte Archéologique de la Gaule 13/5. Arles, Crau, Camargue, Paris 2008.

ROYMANS, N./ZANDSTRA, M.: Indications for rural slavery in the northern provinces, in: Roymans, N./Derks, T. (Hrsg.) Villa Landscapes in the Roman North. Economy, Culture and Lifestyles, Amsterdam 2011, S. 169-174.

RUFFING, Kai: Wirtschaft in der griechisch-römischen Antike, Darmstadt 2012.

Sablayrolles, R./Beyrie, A. (Hrsg.): Carte Archéologique de la Gaule 31/2. Le Comminges (Haute-Garonne), Paris 2006.

SAMSON, R.: Rural Slavery, Inscriptions, Archeology and Marx. A Response to Ramsay MacMullen's "Late Roman Slavery", in: Historia 38/1 (1989), S. 99-110.

SCHÄFER, Ch.: Procuratores, actores und vilici. Zur Leitung landwirtschaftlicher Betriebe im Imperium Romanum, in: HeRz, P./WALDHERR, G. (Hrsg.): Landwirtschaft im Imperium Romanum (Pharos - Studien zur griechischrömischen Antike, Band XIV), St. Katharinen 2001, S. 273-284.

SCHUMACHER, L.: Sklaverei in der Antike. Alltag und Schicksal der Unfreien, München 2001. 
Frankfurter elektronische Rundschau zur Altertumskunde 39 (2019)

Sudi-Guiral, F.: Les servi publici. Actores des cités, in: BerRendOnNer, C. (Hrsg.): Le quotidien municipal dans l'Occident romain, Clermont-Ferrand 2008, S. 405-418.

Thompson, H.: Iron Age and Roman slave-shackles, in: AJ 150 (1993), S. 57-168.

TRÉMENT, F.: Romanisation et développement dans les campagnes des Gaules, in: Ouzoulias, P./Tranoy, L. (Hrsg.): Comment les Gaules devinrent romaines, Paris 2010, S. 159-176.

WALSER, G.: Römische Inschriften der Schweiz, Bd. 2. Nordwest- und Nordschweiz, Bern 1980.

WeEBer, K-W.: Alltag im Alten Rom. Das Landleben, Düsseldorf/Zürich 2000.

WIERSCHOWSKI, L.: Fremde in Gallien - "Gallier" in der Fremde. Die epigraphisch bezeugte Mobilität in, von und nach Gallien vom 1. bis 3. Jh. n. Chr., Stuttgart 2001 . 